\title{
ON A CLASS OF HEREDITARY CROSSED-PRODUCT ORDERS
}

\author{
JOHN S. KAUTA
}

(Communicated by Birge Huisgen-Zimmermann)

Dedicated to the memory of my mom

\begin{abstract}
In this brief note, we revisit a class of crossed-product orders over discrete valuation rings introduced by D. E. Haile. We give simple but useful criteria, which involve only the two-cocycle associated with a given crossedproduct order, for determining whether such an order is a hereditary order or a maximal order.
\end{abstract}

If $R$ is a ring, then $J(R)$ will denote its Jacobson radical, $U(R)$ its group of multiplicative units, and $R^{\#}$ the subset of all the non-zero elements. The terminology used in this paper, if not in [1], can be found in [3]. The book by Reiner [3] is also an excellent source of literature on maximal orders and hereditary orders.

Let $V$ be a discrete valuation ring (DVR), with quotient field $F$, and let $K / F$ be a finite Galois extension, with group $G$, and let $S$ be the integral closure of $V$ in $K$. Let $f \in Z^{2}(G, U(K))$ be a normalized two-cocycle. If $f(G \times G) \subseteq S^{\#}$, then one can construct a "crossed-product" $V$-algebra

$$
A_{f}=\sum_{\sigma \in G} S x_{\sigma}
$$

with the usual rules of multiplication $\left(x_{\sigma} s=\sigma(s) x_{\sigma}\right.$ for all $s \in S, \sigma \in G$ and $x_{\sigma} x_{\tau}=$ $\left.f(\sigma, \tau) x_{\sigma \tau}\right)$. Then $A_{f}$ is associative, with identity $1=x_{1}$, and center $V=V x_{1}$. Further, $A_{f}$ is a $V$-order in the crossed-product $F$-algebra $\Sigma_{f}=\sum_{\sigma \in G} K x_{\sigma}=$ $(K / F, G, f)$.

Two such cocycles $f$ and $g$ are said to be cohomologous over $S$ (respectively cohomologous over $K$ ), denoted by $f \sim_{S} g$ (respectively $f \sim_{K} g$ ), if there are elements $\left\{c_{\sigma} \mid \sigma \in G\right\} \subseteq U(S)$ (respectively $\left\{c_{\sigma} \mid \sigma \in G\right\} \subseteq K^{\#}$ ) such that $g(\sigma, \tau)=c_{\sigma} \sigma\left(c_{\tau}\right) c_{\sigma \tau}^{-1} f(\sigma, \tau)$ for all $\sigma, \tau \in G$. Following [1, let $H=\{\sigma \in G \mid$ $\left.f\left(\sigma, \sigma^{-1}\right) \in U(S)\right\}$. Then $H$ is a subgroup of $G$. On $G / H$, the left coset space of $G$ by $H$, one can define a partial ordering by the rule $\sigma H \leq \tau H$ if $f\left(\sigma, \sigma^{-1} \tau\right) \in U(S)$. Then " $\leq$ " is well-defined and depends only on the cohomology class of $f$ over $S$. Further, $H$ is the unique least element. We call this partial ordering on $G / H$ the graph of $f$.

Such a setup was first formulated by Haile in [1, with the assumption that $S$ is unramified over $V$, wherein, among other things, conditions equivalent to such orders being maximal orders were considered. This is the class of crossed-product orders we shall study in this paper, always assuming that $S$ is unramified over $V$. We emphasize the fact that, since we do not require that $f(G \times G) \subseteq U(S)$, this

Received by the editors August 7, 2010 and, in revised form, September 2, 2011.

2010 Mathematics Subject Classification. Primary 16H10, 16S35, 16E60, 13F30. 
theory constitutes a drastic departure from the classical theory of crossed-product orders over DVRs, such as can be found in [2].

Let us now fix additional notation to be used in the rest of the paper, most of it borrowed from [1] as before. If $M$ is a maximal ideal of $S$, let $D_{M}$ be the decomposition group of $M$, let $K_{M}$ be the decomposition field, and let $S_{M}$ be the localization of $S$ at $M$. The two-cocycle $f: G \times G \mapsto S^{\#}$ yields a two-cocycle $f_{M}: D_{M} \times D_{M} \mapsto S_{M}^{\#}$, determined by the restriction of $f$ to $D_{M} \times D_{M}$ and the inclusion of $S^{\#}$ in $S_{M}^{\#}$. Then $A_{f_{M}}=\sum_{\sigma \in D_{M}} S_{M} x_{\sigma}$ is a crossed-product order in $\Sigma_{f_{M}}=\sum_{\sigma \in D_{M}} K x_{\sigma}=\left(K / K_{M}, D_{M}, f_{M}\right)$. In addition, we can obtain a twist of $f$, described in [1, pp. 137-138] and denoted by $\tilde{f}$, which depends on the choice of a maximal ideal $M$ of $S$, and the choice of a set of coset representatives of $D_{M}$ in $G$. We also define $F: G \times G \mapsto S^{\#}$ by $F(\sigma, \tau)=f\left(\sigma, \sigma^{-1} \tau\right)$ for $\sigma, \tau \in G$. While $\tilde{f}$ is a two-cocycle, $F$ is not.

If $B$ is a $V$-order of $\Sigma_{f}$ containing $A_{f}$, then by [1, Proposition 1.3], $B=A_{g}=$ $\sum_{\sigma \in G} S y_{\sigma}$ for some two-cocycle $g: G \times G \mapsto S^{\#}$, with $g \sim_{K} f$. Moreover, the proof of [1, Proposition 1.3] shows that $y_{\sigma}=k_{\sigma} x_{\sigma}$ for some $k_{\sigma} \in K^{\#}$, with $k_{1}=1$, whence $g$ is also a normalized two-cocycle.

We begin with a technical result.

Sublemma. Let $\tau \in G$. If $I_{\tau}=\prod_{f\left(\tau, \tau^{-1}\right) \notin M} M$, where $M$ denotes a maximal ideal of $S$, then $I_{\tau}^{\tau^{-1}}=I_{\tau^{-1}}$.

Proof. We have

$$
I_{\tau}^{\tau^{-1}}=\prod_{f\left(\tau, \tau^{-1}\right) \notin M} M^{\tau^{-1}}=\prod_{f^{-1}}=\prod_{\left(\tau, \tau^{-1}\right) \notin M^{\tau^{-1}}}^{\tau^{-1}}=\underset{f\left(\tau^{-1}, \tau\right) \notin M^{\tau^{-1}}}{\prod_{\tau^{-1}}}
$$

Theorem. The crossed-product order $A_{f}$ is hereditary if and only if $f\left(\tau, \tau^{-1}\right) \notin M^{2}$ for all $\tau \in G$ and every maximal ideal $M$ of $S$.

Proof. The theorem obviously holds if $H=G$, in which case $A_{f}$ is an Azumaya algebra over $V$, so let us assume from now on that $H \neq G$.

Suppose $A_{f}$ is hereditary. First, assume $A_{f}$ is a maximal order and $S$ is a DVR. Let $v$ be the valuation corresponding to $S$ with value group $\mathbb{Z}$. Then by 1 , Theorem 2.3], $H$ is a normal subgroup of $G$ and $G / H$ is cyclic. Further, there exists $\sigma \in G$ such that $v\left(f\left(\sigma, \sigma^{-1}\right)\right) \leq 1, G / H=\langle\sigma H\rangle$, and the graph of $f$ is the chain $H \leq \sigma H \leq \sigma^{2} H \leq \cdots \leq \sigma^{m-1} H$, where $m=|G / H|$. Choose $j$ maximal such that $1 \leq j \leq m-1$ and $v\left(f\left(\sigma^{i}, \sigma^{-i}\right)\right) \leq 1 \forall 1 \leq i \leq j$. We always have $\sigma H \leq \sigma^{-j} H$; but if $j<m-1$, then we also have $\sigma^{j} H \leq \sigma^{j+1} H$. Hence if $j<m-1$, then, from the cocycle identity $f^{\sigma^{j}}\left(\sigma, \sigma^{-j} \sigma^{-1}\right) f\left(\sigma^{j}, \sigma^{-j}\right)=f\left(\sigma^{j}, \sigma\right) f\left(\sigma^{j+1}, \sigma^{-j} \sigma^{-1}\right)$, we conclude that $v\left(f\left(\sigma^{i}, \sigma^{-i}\right)\right) \leq 1 \forall 1 \leq i \leq j+1$, a contradiction. So we must have $j=m-1$, so that $v\left(f\left(\sigma^{i}, \sigma^{-i}\right)\right) \leq 1 \forall 1 \leq i \leq m-1$. If $\tau$ is an arbitrary element of $G$, then $\tau=\sigma^{i} h$ for some $h \in H$ and some integer $i, 0 \leq i \leq m-1$. Therefore, by [1, Lemma 3.6], $v\left(f\left(\tau, \tau^{-1}\right)\right)=v\left(F\left(\sigma^{i} h, 1\right)\right)=v\left(F\left(\sigma^{i}, 1\right)\right)=v\left(f\left(\sigma^{i}, \sigma^{-i}\right)\right) \leq 1$; that is, $f\left(\tau, \tau^{-1}\right) \notin J(S)^{2}$.

We maintain the assumption that $A_{f}$ is a maximal order, but we now drop the condition that $S$ is a DVR. By [1, Theorem 3.16], there exists a twist of $f$, say $\tilde{f}$, such that $f \sim_{S} \tilde{f}$. By [1, Corollary 3.11], for any maximal ideal $M$ of $S, A_{f_{M}}$ is a maximal order in $\Sigma_{f_{M}}$; hence $f_{M}\left(\tau, \tau^{-1}\right) \notin M^{2} \forall \tau \in D_{M}$ by the preceding 
paragraph. Therefore, from the manner in which $\tilde{f}$ is constructed from $f$, we infer that $\tilde{f}\left(\tau, \tau^{-1}\right) \notin M^{2} \forall \tau \in G$ and any maximal ideal $M$ of $S$, and thus $f\left(\tau, \tau^{-1}\right) \notin M^{2} \forall \tau \in G$ and every maximal ideal $M$ of $S$, since $f \sim_{S} \tilde{f}$.

If $A_{f}$ is not a maximal order, then it is the intersection of finitely many maximal orders, say $A_{f_{1}}, A_{f_{2}}, \ldots, A_{f_{l}}$. Note that

$$
A_{f_{i}}=\sum_{\tau \in G} S y_{\tau}^{(i)}=\sum_{\tau \in G} S k_{\tau}^{(i)} x_{\tau}
$$

for some $k_{\tau}^{(i)} \in K$. Fix a $\sigma \in G$, and a maximal ideal $N$ of $S$. Let $v_{N}$ be the valuation corresponding to $N$, with value group $\mathbb{Z}$. Since

$$
S=\bigcap_{i=1}^{l} S k_{\sigma}^{(i)},
$$

there exists $i_{0}$ such that $v_{N}\left(k_{\sigma}^{\left(i_{0}\right)}\right)=0$. Let $g=f_{i_{0}}$ and, for $\tau \in G$, let $k_{\tau}=k_{\tau}^{\left(i_{0}\right)}$ and $y_{\tau}=y_{\tau}^{\left(i_{0}\right)}$, so that $A_{g}=\sum_{\tau \in G} S k_{\tau} x_{\tau}=\sum_{\tau \in G} S y_{\tau}$. By [1, Proposition 3.1], $J\left(A_{f}\right)=\sum_{\tau \in G} I_{\tau} x_{\tau}$ and $J\left(A_{g}\right)=\sum_{\tau \in G} J_{\tau} y_{\tau}$, where

$$
I_{\tau}=\prod_{f\left(\tau, \tau^{-1}\right) \notin M} M \quad \text { and } \quad J_{\tau}=\prod_{g\left(\tau, \tau^{-1}\right) \notin M} M,
$$

and $M$ denotes a maximal ideal of $S$. Since $A_{f}$ is a hereditary $V$-order in $\Sigma_{f}$ and $A_{f} \subseteq A_{g} \subseteq \Sigma_{f}$, we have $J\left(A_{g}\right) \subseteq J\left(A_{f}\right)$, from which we conclude that $J_{\sigma^{-1}} y_{\sigma^{-1}} \subseteq$ $I_{\sigma^{-1}} x_{\sigma^{-1}}$ and so $J_{\sigma^{-1}} k_{\sigma^{-1}} \subseteq I_{\sigma^{-1}}$. We have $y_{\sigma^{-1}} J_{\sigma} y_{\sigma}=k_{\sigma^{-1}} x_{\sigma^{-1}} J_{\sigma} k_{\sigma} x_{\sigma}=$ $J_{\sigma^{-1}} k_{\sigma^{-1}} x_{\sigma^{-1}} k_{\sigma} x_{\sigma} \subseteq I_{\sigma^{-1}} x_{\sigma^{-1}} k_{\sigma} x_{\sigma}=\sigma^{-1}\left(k_{\sigma}\right) I_{\sigma^{-1}} f\left(\sigma^{-1}, \sigma\right)=\left(k_{\sigma} I_{\sigma} f\left(\sigma, \sigma^{-1}\right)\right)^{\sigma^{-1}}$. On the other hand, $y_{\sigma^{-1}} J_{\sigma} y_{\sigma}=J_{\sigma}^{\sigma^{-1}} g\left(\sigma^{-1}, \sigma\right)=J_{\sigma^{-1}} g\left(\sigma^{-1}, \sigma\right)$. Since $A_{g}$ is a maximal order and therefore $g\left(\sigma^{-1}, \sigma\right) \notin M^{2}$ for every maximal ideal $M$ of $S$, we see that $J_{\sigma^{-1}} g\left(\sigma^{-1}, \sigma\right)=J(V) S$ and so $y_{\sigma^{-1}} J_{\sigma} y_{\sigma}=J(V) S$. Therefore $J(V) S \subseteq k_{\sigma} I_{\sigma} f\left(\sigma, \sigma^{-1}\right)$. Since $v_{N}\left(k_{\sigma}\right)=0$, we conclude that $f\left(\sigma, \sigma^{-1}\right) \notin N^{2}$, and so $f\left(\tau, \tau^{-1}\right) \notin M^{2} \forall \tau \in G$ and any maximal ideal $M$ of $S$.

Conversely, suppose that $f\left(\tau, \tau^{-1}\right) \notin M^{2}$ for every maximal ideal $M$ of $S$ and every $\tau \in G$. Let $B=O_{l}\left(J\left(A_{f}\right)\right)$, the left order of $J\left(A_{f}\right)$; that is, $B=\left\{x \in \Sigma_{f} \mid\right.$ $\left.x J\left(A_{f}\right) \subseteq J\left(A_{f}\right)\right\}$. Since $\Sigma_{f} \supseteq B \supseteq A_{f}, B=\sum_{\tau \in G} S k_{\tau} x_{\tau}$, for some $k_{\tau} \in K^{\#}$. For each $\tau \in G$, we have $S \subseteq S k_{\tau}$, and we will now show that $S=S k_{\tau}$. As above, write $J\left(A_{f}\right)=\sum I_{\tau} x_{\tau}$, with $I_{\tau}=\prod M$, where the product is taken over all maximal ideals $M$ of $S$ for which $f\left(\tau, \tau^{-1}\right) \notin M$. Observe that $J(V) S=$ $I_{1} \supseteq k_{\tau} x_{\tau} I_{\tau^{-1}} x_{\tau^{-1}}=k_{\tau} I_{\tau^{-1}}^{\tau} f\left(\tau, \tau^{-1}\right)=k_{\tau} I_{\tau} f\left(\tau, \tau^{-1}\right)$. Since $f\left(\tau, \tau^{-1}\right) \notin M^{2}$ for every maximal ideal $M$ of $S$, we must have $I_{\tau} f\left(\tau, \tau^{-1}\right)=J(V) S$, and so $J(V) S \supseteq$ $k_{\tau} J(V) S \supseteq J(V) S$ and thus $S=S k_{\tau}$, as desired. This shows that $O_{l}\left(J\left(A_{f}\right)\right)=A_{f}$ and $A_{f}$ is hereditary.

Not only can this criterion enable one to rapidly determine whether or not the crossed-product order $A_{f}$ is hereditary, the utility of the theorem above is now demonstrated by the ease with which the following corollaries of it are obtained.

Corollary 1. The crossed-product order $A_{f}$ is hereditary if and only if $f(\tau, \gamma) \notin M^{2}$ for all $\tau, \gamma \in G$ and every maximal ideal $M$ of $S$.

Proof. This follows from the cocycle identity $f^{\tau}\left(\tau^{-1}, \tau \gamma\right) f(\tau, \gamma)=f\left(\tau, \tau^{-1}\right)$. 
In other words, the order $A_{f}$ is hereditary if and only if the values of the twococycle $f$ are all square-free.

Since $A_{f}$ is a maximal order if and only if it is hereditary and primary, by combining our result and results in [1, we immediately have the following.

Corollary 2. Given a crossed-product order $A_{f}$,

(1) it is a maximal order if and only if for every maximal ideal $M$ of $S$, $f\left(\tau, \tau^{-1}\right) \notin M^{2}$ for all $\tau \in G$, and there exists a set of right coset representatives $g_{1}, g_{2}, \ldots, g_{r}$ of $D_{M}$ in $G$ (i.e., $G$ is the disjoint union $\bigcup_{i} D_{M} g_{i}$ ) such that for all $i, f\left(g_{i}, g_{i}^{-1}\right) \notin M$.

(2) if $S$ is a DVR, then it is a maximal order if and only if $f\left(\tau, \tau^{-1}\right) \notin J(S)^{2}$ for all $\tau \in G$.

Proof. In either case, the primarity of $A_{f}$ is guaranteed by [1, Theorem 3.2] (see also [1, Proposition 2.1(b)] when $S$ is a DVR).

The Theorem above can readily be put to effective use with the crossed-product orders in [1, §4], for example. In that section, all the crossed-product orders involved are primary orders, and the two-cocycles are given in tabular form, with the values factorized into primes of $S$. Using our criterion, it now becomes a straightforward process to determine which of those orders are maximal orders and which are not, by simply consulting, in each case, the given table of values for the two-cocycle; the table whose entries are all square-free represents a maximal order. This determination can be made with little effort! In fact, if one knows that the crossed-product order $A_{f}$ is a primary order, then determining whether or not it is a maximal order could even be easier, as the following result shows.

Corollary 3. Suppose the crossed-product order $A_{f}$ is primary. Then it is a maximal order if and only if there exists a maximal ideal $M$ of $S$ such that $f\left(\tau, \tau^{-1}\right) \notin$ $M^{2}$ for all $\tau \in D_{M}$.

Proof. This follows from [1, Corollary 3.11 and Proposition 2.1(b)].

Let $L$ be an intermediate field of $F$ and $K$, let $G_{L}$ be the Galois group of $K$ over $L$, let $U$ be a valuation ring of $L$ lying over $V$, and let $T$ be the integral closure of $U$ in $K$. Then one can obtain a two-cocycle $f_{L, U}: G_{L} \times G_{L} \mapsto T^{\#}$ from $f$ by restricting $f$ to $G_{L} \times G_{L}$ and embedding $S^{\#}$ in $T^{\#}$. As before, $A_{f_{L}, U}=\sum_{\tau \in G_{L}} T x_{\tau}$ is a $U$-order in $\Sigma_{f_{L, U}}=\sum_{\tau \in G_{L}} K x_{\tau}=\left(K / L, G_{L}, f_{L, U}\right)$.

Corollary 4. Suppose the crossed-product order $A_{f}$ is hereditary. Then $A_{f_{L, U}}$ is a hereditary order in $\Sigma_{f_{L, U}}$ for each intermediate field $L$ of $F$ and $K$ and for every valuation ring $U$ of $L$ lying over $V$.

This leads to the following.

Corollary 5. Suppose the crossed-product order $A_{f}$ is hereditary. Then $A_{f_{M}}$ is a maximal order in $\Sigma_{f_{M}}$ for each maximal ideal $M$ of $S$.

Proof. The order $A_{f_{M}}$ is always primary, by [1, Proposition 2.1(b)].

The following example illustrates two limitations of our theory, however.

Example. We give two crossed-product orders $A_{f_{1}}$ and $A_{f_{2}}$ with $f_{1} \sim_{K} f_{2}$ and the graphs of $f_{1}$ and $f_{2}$ identical, but $A_{f_{1}}$ is hereditary while $A_{f_{2}}$ is not. Also, we give an example to demonstrate that the converse of Corollary 5 does not always hold. 
Let $F=\mathbb{Q}(x)$, and let $K=\mathbb{Q}(i)(x)$. Then the Galois group $G=\langle\sigma\rangle$ is a group of order two, where $\sigma$ is induced by the complex conjugation on $\mathbb{Q}(i)$. If $V=\mathbb{Q}[x]_{\left(x^{2}+1\right)}$, then $S$ has two maximal ideals, namely $M_{1}=(x+i) S$ and $M_{2}=$ $(x-i) S$, and $D_{M_{1}}=D_{M_{2}}=\{1\}$. Let $f_{1}, f_{2}: G \times G \mapsto S^{\#}$ be two-cocycles defined by $f_{j}(1,1)=f_{j}(1, \sigma)=f_{j}(\sigma, 1)=1$ and $f_{1}(\sigma, \sigma)=\left(x^{2}+1\right) x, f_{2}(\sigma, \sigma)=\left(x^{2}+1\right)^{2} x$.

Then $f_{1} \sim_{K} f_{2}$, and the subgroup of $G$ associated with either cocycle is $H=\{1\}$, so that the graphs of $f_{1}$ and $f_{2}$ are identical. Clearly, $A_{f_{1}}$ is hereditary but $A_{f_{2}}$ is not. We conclude that the property that a crossed-product order $A_{f}$ is hereditary is not an intrinsic property of the graph of $f$.

Also, if we set $f=f_{2}$, we see that $A_{f_{M}}=S_{M}$ for each maximal ideal $M$ of $S$, and therefore $A_{f_{M}}$ is a maximal order in $\Sigma_{f_{M}}=K$ for each maximal ideal $M$ of $S$, and yet $A_{f}$ is not even hereditary (cf. [1, Corollary 3.11] and [2, Theorem 1]). This is the case because $A_{f}$ is not primary, and also because $f(G \times G) \nsubseteq U(S)$.

\section{REFERENCES}

1. D. E. Haile, Crossed-product orders over discrete valuation rings, J. Algebra 105 (1987), 116-148. MR0871749(88b:16013)

2. M. Harada, Some criteria for hereditarity of crossed products, Osaka J. Math. 1 (1964), 69-80. MR0174584(30:4785)

3. I. Reiner, Maximal Orders, Academic Press, London, 1975. MR0393100 (52:13910)

Department of Mathematics, Faculty of Science, Universiti Brunei Darussalam, Bandar Seri Begawan, BE1410, Brunei

E-mail address: john.kauta@ubd.edu.bn 